\title{
An Energy Efficient Cluster-heads Re-usability Mechanism for Wireless Sensor Networks
}

\author{
Syed Kamran Haider ${ }^{1}$, Muhammad Ali Jamshed ${ }^{2}$, Aimin Jiang ${ }^{3}$, and Haris Pervaiz ${ }^{4}$ \\ ${ }^{1,3}$ College of Internet of Things Engineering, Hohai University Changzhou, China \\ ${ }^{2}$ Institute of Communication Systems (ICS), Home of 5G Innovation Centre (5GIC), University of Surrey, UK \\ ${ }^{4}$ School of Computing and Communications, Lancaster University, UK \\ Email: kamranhaider85@yahoo.com,m.jamshed@surrey.ac.uk,jiangam@hhuc.edu.cn, h.b.pervaiz@lancaster.ac.uk
}

\begin{abstract}
Wireless sensor networks (WSNs) are formed using a cluster of sensor nodes (SNs), deployed randomly to perform sensing operations in an area under observation. Due to the unavailability of an external power source, the energy efficiency considered as one of the critical issues in WSNs. Selection of a sensor node (SN) from a wireless sensor network (WSN) cluster to serve as an aggregator or cluster head (CH), considered as an efficient method to increase the lifetime of wireless sensor network (WSN). In this paper, we have proposed an energy efficient CH selection scheme for WSN, to enhance the lifetime and average residual energy of a single WSN cluster. The proposed strategy nominates a group $\mathrm{SNs}$ as $\mathrm{CHs}$, based on their channel condition with the base station (BS) and their residual energy. The proposed algorithm is helpful in solving the problem of unbalanced energy consumption in WSNs. Furthermore, the mechanism of using mobile sink during the hand-off stage helps to overcome the delay in data transmission. Moreover, the incorporation of energy harvesting significantly increases the lifetime of WSN. In comparison to a state-of-art technique available in the literature, our scheme shows a $33 \%$ increase in lifetime and presents a steady decrease in residual energy for the same rounds of data transmission.
\end{abstract}

Index Terms-Wireless sensor network (WSN), lifetime, cluster head selection, average residual energy.

\section{INTRODUCTION}

$\mathrm{W}$ IRELESS sensor networks (WSNs) offers a vast range of advantages to many applications by lowering their cost and reducing their overall power consumption [1]. These additive advantages of wireless sensor network (WSN) have increased their use in many fields including advanced agriculture [2], [3] and many other, related to the automatic irrigation system for farmland [4], to improve the yield of crops. The integration of WSN in the internet of things (IoT) can be used to generate an early warning of earthquake [5]. In this context, the WSN provides a wide range of applications and considered as one of key enabling technology for IoT. A WSN is an arrangement of randomly distributed devices, i.e. sensor nodes (SNs), which are interconnected having sensing capabilities to communicate and share data with some special features [6]. These SNs are used for multimedia applications by installing a CMOS device, e.g. camera, on sensor node (SN) [7]. Each SN is equipped with limited resources and thrown in terrain with an unconditional climate having a pre-configured set of tasks, that it needs to perform. Each of deployed SN in WSN cluster faces a considerable energy issue and needs to manipulate the power consumption to increase their lifetime and make sure that they perform all the allocated tasks.

Diverse solutions are presented in the literature to allow each SN in WSN to utilise their limited resources efficiently. Many energy efficient scheduling techniques are available in the literature [8], to accommodate each SN to perform well under confined power resources. Authors in [9] proposed an idea of multicore energy efficient scheduling with energy harvesting for wireless multimedia sensor network (WMSNs), by utilising lightweight processors (LWP) to manifold multiple tasks practically, without compromising on deadline. In [10] a routing protocol based on spanning tree technique using the hybrid multi-hop partition clustering, is presented to enhance the lifetime of the WSN.

\section{A. Related Work}

A plethora of research work has been done by the research community to enhance the energy efficiency of WSN. Deploying each SN in WSN using an effective deployment scheme is considered as one of the critical methodologies to improve the energy efficiency of WSN. In connection, clustering can be utilized while considering the dense deployment of SNs to maintain an acceptable average energy consumption. In [11] a clustering methodology has been introduced that helps the network to recuperate SNs from the futile gateway without considering, re-clustering or close down of the system to increase the overall system efficiency. Furthermore, in [12] the authors propose a constrained coverage (CC) algorithm which introduces two virtual forces to ensure that each cluster node has at least K-Neighbors, but this scheme may result in a low network coverage area and a decrease in lifetime of SNs. Similarly, in [13] the concept of virtual force is utilized for clustering, but the mechanism may lead to an unstable lifetime of WSN.

The selection of a cluster head $(\mathrm{CH})$ in a WSN cluster can be used to prolong the network lifetime and to maximize the communication range. Low-energy adaptive clustering hierarchy (LEACH) [14], is considered as one of the main clustering algorithms to enhance the lifetime of a WSN cluster. 
An acceptable amount of research work is available in the literature that exploits the LEACH protocol to increase the energy efficiency of WSN [15]. In [16] a two-step optimized zone based energy efficient protocol (OZEEP) has been proposed, which incorporates the genetic fuzzy systems (GFS) to optimize the clustering and the selection of cluster heads (CHs) in WSN. Authors in [17] suggested a routing protocol based on a novel energy-aware hierarchical cluster (NEAHC) to select $\mathrm{CHs}$ by introducing threshold schemes. An energy efficient adaptive selection of $\mathrm{CH}$ for wireless sensor networks (WSNs) has been proposed in [18], that uses the dissipated and residual energy levels in the modified version of $\mathrm{LEACH}$ protocol to provide a balance energy efficiency.

A dynamic cluster head selection method (DCHSM) for WSN has been introduced in [19], that dynamically selects a $\mathrm{CH}$ based on their residual energy and dynamically changes the $\mathrm{CH}$ after the death of the previous $\mathrm{CH}$. In [20] the authors use the approach of hesitant fuzzy for $\mathrm{CH}$ selection by analysing their distance from the centre of the cluster and their range from the base station (BS) location. Similarly, in [21] the concept of nominating a $\mathrm{CH}$ based on their position on a WSN cluster is combined with their energy consumption while assuming a uniform distribution of SNs. In connection, the authors in [22] connect the location-based approach with balanced energy consumption to select a $\mathrm{CH}$. Furthermore, the concept of spare $\mathrm{CH}$ is introduced in [23], while considering a hybrid key management mechanism.

\section{B. Our Contributions}

In this paper, we have proposed an energy efficient $\mathrm{CH}$ selection scheme to optimize the overall lifetime and average energy consumption of a WSN cluster. The proposed technique significantly increases the lifetime of WSN and provides a solution to the problem of unbalanced energy consumption in the WSNs. The main highlighted contributions of the paper are as follows:

- An energy efficient scheme has been proposed that equips the BS with a group of CHs, selected from a WSN cluster.

- The idea of the mobile sink has been utilized to decrease the delay and to efficiently cater to the low power state of the $\mathrm{CH}$ during the hand-off stage.

- To prolong the network survival time, each $\mathrm{CH}$ changes its state to sleep mode and perform energy harvesting after reaching the hand-off threshold level.

- We are incorporating the use of harvesting energy to enhance the reusability of each $\mathrm{CH}$ node, enduring the sleep mode.

The remaining part of the paper is organized as follows: Section 2 explained the system model and the proposed scheme in detail. Section 3 shows a comparison of the proposed methodology with a technique available in literature through some simulations. Finally, we conclude the paper in Section 4. Moreover, in Table I, symbols used throughout the article has been listed to provide ease to the readers.
TABLE I

SYMBOLS USED THROUGHOUT THE PAPER

\begin{tabular}{c|l}
\hline \hline Symbol & \multicolumn{1}{|c}{ Meaning } \\
\hline$\delta$ & Hand-off threshold \\
\hline$\beta$ & Residual energy threshold \\
\hline$\alpha$ & Channel gain threshold \\
\hline$\gamma$ & SNR threshold \\
\hline$g_{n}$ & Gain of user $n$ \\
\hline$P_{n}$ & Received power of user $n$ \\
\hline$d$ & BS location from WSN Cluster \\
\hline$\tau$ & path loss exponent \\
\hline$P t_{n}$ & Transmit power \\
\hline$R s_{n}$ & Residual energy of user $n$ \\
\hline
\end{tabular}

\section{SYSTEM MODEL AND PROPOSED SCHEME}

\section{A. System Model}

We have assumed a single cluster of WSN, pictorially expressed in Fig. 1. The cluster of a WSN consists of $N$ number of SNs that are generated randomly and deployed uniformly. A BS is located at a distance $d$ from the WSN cluster. A Rayleigh fading channel has been assumed between each transmitter and receiver pair. The following equation can be used to calculate the path-loss between each transmitter and receiver in $\mathrm{dB}$ :

$$
\mathrm{PL}=\mathrm{FSPL}+10 \tau \log _{10}(s)+X_{\zeta},
$$

where, $s$ is the distance between the receiver and the transmitter, $\tau$ is the path-loss exponent, $X_{\zeta}$ incorporates the large scale signal variation, and FSPL is the free space path loss. The FSPL can be calculated using the following equation

$$
\mathrm{FSPL}=20 \log _{10}\left(\frac{4 \pi f}{c}\right)
$$

where, $f$ is the carrier frequency and $c$ is the speed of light. Thus, the received power $P_{n}$ of $\mathrm{SN} n$ at the receiver end is calculated using the following equation:

$$
P_{n}=\mathrm{PL} * P t_{n} * g_{n}^{-\tau},
$$

where, $P t_{n}$ is the transmit power of $\mathrm{SN} n$ and $g_{n}$ is the channel gain of the same SN. A series of SNs are selected by the $\mathrm{BS}$, to nominate them as a $\mathrm{CH}$. The selected $\mathrm{SNs}$ is then dynamically assigned to a numerical rank value. The $\mathrm{SN}$ with the highest rank is selected as the $\mathrm{CH}$. Moreover, if the $R_{n}$ of the selected $\mathrm{CH}$ node drop below the hand-off threshold $\delta$, a hand-off procedure is initiated by cooperating with a nearby mobile sink, this cooperation reduces the delay in the data transmission between the SNs and the BS. The $\mathrm{SN}$, which handover its duty as a $\mathrm{CH}$, changes its state from active to sleep and starts harvesting energy to be renominated as $\mathrm{CH}$. In this experimental setup, we have assumed that the SNs changing their state, have a redundant node, which is considered while SNs deployment. We have defined one round 


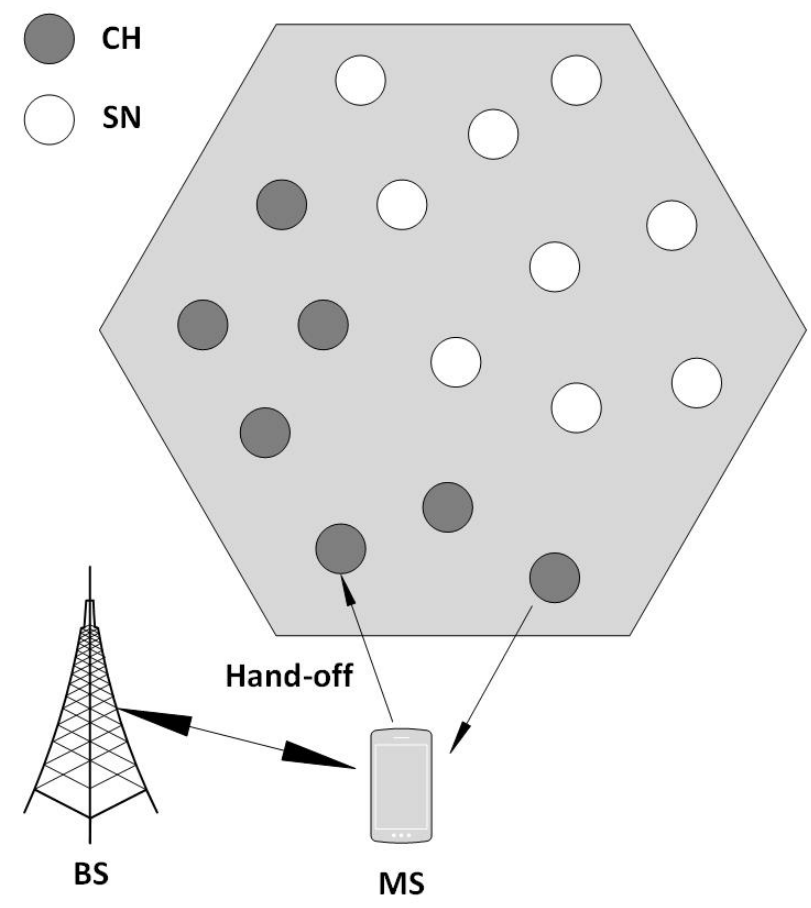

Fig. 1. An illustration of a WSN cluster having SNs with selected $\mathrm{CHs}$ and the hand-off procedure using a nearby mobile sink.

as the time taken by the packet to be successfully received by the BS, transmitted from the $\mathrm{CH}$. In connection, the time taken to perform a hand-off is also considered as one round. Furthermore, we assume that for each round the overall energy consumption is the same. The energy consumption in each round $E_{r n d}$ can be calculated using the following equation:

$$
E_{r n d}=t\left(N * \mathrm{PL}+N * \theta * D_{S-C}+\theta * D_{C-B}\right),
$$

where, $t$ is the total taken by each round, $D_{S-C}$ is the distance between the $\mathrm{SNs}$ and the current $\mathrm{CH}, D_{C-B}$ is the distance between the current $\mathrm{CH}$ and the $\mathrm{BS}$ and $\theta$ is the total re-transmission losses. Therefore the lifetime of WSN is calculated using the following equation:

$$
L_{r}=\frac{E_{t}}{E_{r n d}},
$$

where, $E_{t}$ is the total initial energy of the network and $L_{r}$ is the total number of successful rounds during the life cycle of WSN.

\section{B. Proposed Scheme}

An energy efficient approach to select a $\mathrm{CH}$ for a WSN is presented in this paper. The proposed approach exploits the use of energy harvesting to increase the overall lifetime of a WSN. A Rayleigh fading channel has been considered between each $\mathrm{CH}$ and the BS. A group of SNs are nominated by the $\mathrm{BS}$ to be considered as $\mathrm{CHs}$, if they satisfy the channel gain threshold $\alpha$ and residual energy threshold $\beta$, respectively. A list of nominated $\mathrm{CHs}$ by the BS are further categorized based on their channel gain $g_{n}$ and residual energy $R_{n}$ and are tagged with a unique identification i.e. $\operatorname{rank}=1, \ldots, r_{n}$, in a descending order. The residual energy at the BS for each $\mathrm{CH}$ is calculated using their $P_{n}$, i.e. we have assumed that $P_{n}$ and $R s_{n}$ have a good correlation. Algorithm 1 provides an insight into the proposed scheme. The proposed algorithm analysis the pilot signal received by each $\mathrm{SN}$ and use $N, g_{n}$, $P_{n}$, and $R s_{n}$ as its main input. Moreover, the algorithm works in the following four stages:

- In stage 1, the algorithm compares the received power $P_{n}$ of each SN with the SNR threshold $\gamma$. The SNs, that satisfies the first condition is further analysed using their channel gain and residual energy values. Again the SNs that satisfies the second condition, are nominated as $\mathrm{CHs}$.

- In stage 2 of the algorithm, the nominated CHs are tagged using a unique identification, i.e. rank and are labelled in descending order, based on their residual energy and channel gain.

- The selection of a $\mathrm{CH}$ for WSN is made at stage 3. The nominated SNs with the highest rank is selected as a $\mathrm{CH}$.

- If the residual energy of the selected $\mathrm{CH}$ drops below the hand-off threshold $\delta$, a hand-off process is initiated by the BS using the cooperation of nearby mobile sink. For the experiment, we have assumed that a mobile sink is always available during the hand-off stage.

After the hand-off stage, the SN changes its state to sleep mode and starts to harvest energy using the installed harvesting circuit. Furthermore, the algorithm runs in a recursive manner till the battery drainage of each SN or the unavailability of a $\mathrm{SN}$ to perform as $\mathrm{CH}$ for WSN. This recursive nature of the algorithm running at the BS helps to re-analyse the SNs performing energy harvesting.

\section{Complexity Analysis of Proposed Algorithm}

In this section, we have calculated the computational complexity of our proposed, $\mathrm{CH}$ selection algorithm, using Algorithm 1. For the input, the number of sensors $N$ has no complexity because of its independent nature. The remaining inputs $g_{n}, P_{n}$, and $R s_{n}$ respectively has a complexity of $\mathcal{O}(N)$, therefore the total complexity of input becomes $\mathcal{O}(N)$. To make the complexity analysis simpler, we have calculated the complexity of each step separately, as shown in Algorithm 1. Starting from step 1, we have a for-loop that performs its operation between line 3 and 9 of the algorithm and has a complexity of $\mathcal{O}(N)$. Inside this forloop, we have a different set of operations having a maximum complexity of $\mathcal{O}(2)$. The output at this stage has a complexity of $\mathcal{O}(N)$. Using this detailed step by step analysis, the overall complexity of step 1 is $\mathcal{O}(N)$. Secondly, for step 2 we have two nested for-loops having a complexity of $\mathcal{O}(N)$ and $\mathcal{O}(N-1)$ respectively. Inside these, for loops, the maximum complexity is $\mathcal{O}(2)$. Therefore the overall complexity of step 2 is $\mathcal{O}\left(N^{2}\right)$. Thirdly, for step 3, again we have a for-loop having a similar complexity of $\mathcal{O}(N)$, because of its dependency on $N$ with inside operations having a maximum complexity of $\mathcal{O}(1)$. The complexity at this stage is $\mathcal{O}(N)$. For the last step of the algorithm, we have a for-loop having an inside operation 
that reconsiders the step 3, which makes the complexity of this step as $\mathcal{O}\left(N^{2}\right)$. Our proposed algorithm has a complexity of $2^{\text {nd }}$ order polynomial, i.e. $\mathcal{O}\left(N^{2}\right)$, which can be explained further using the following equation:

$$
\mathcal{O}\left(N^{2}\right)=\mathcal{O}(N)+\mathcal{O}\left(N^{2}\right)+\mathcal{O}(N)+\mathcal{O}\left(N^{2}\right)
$$

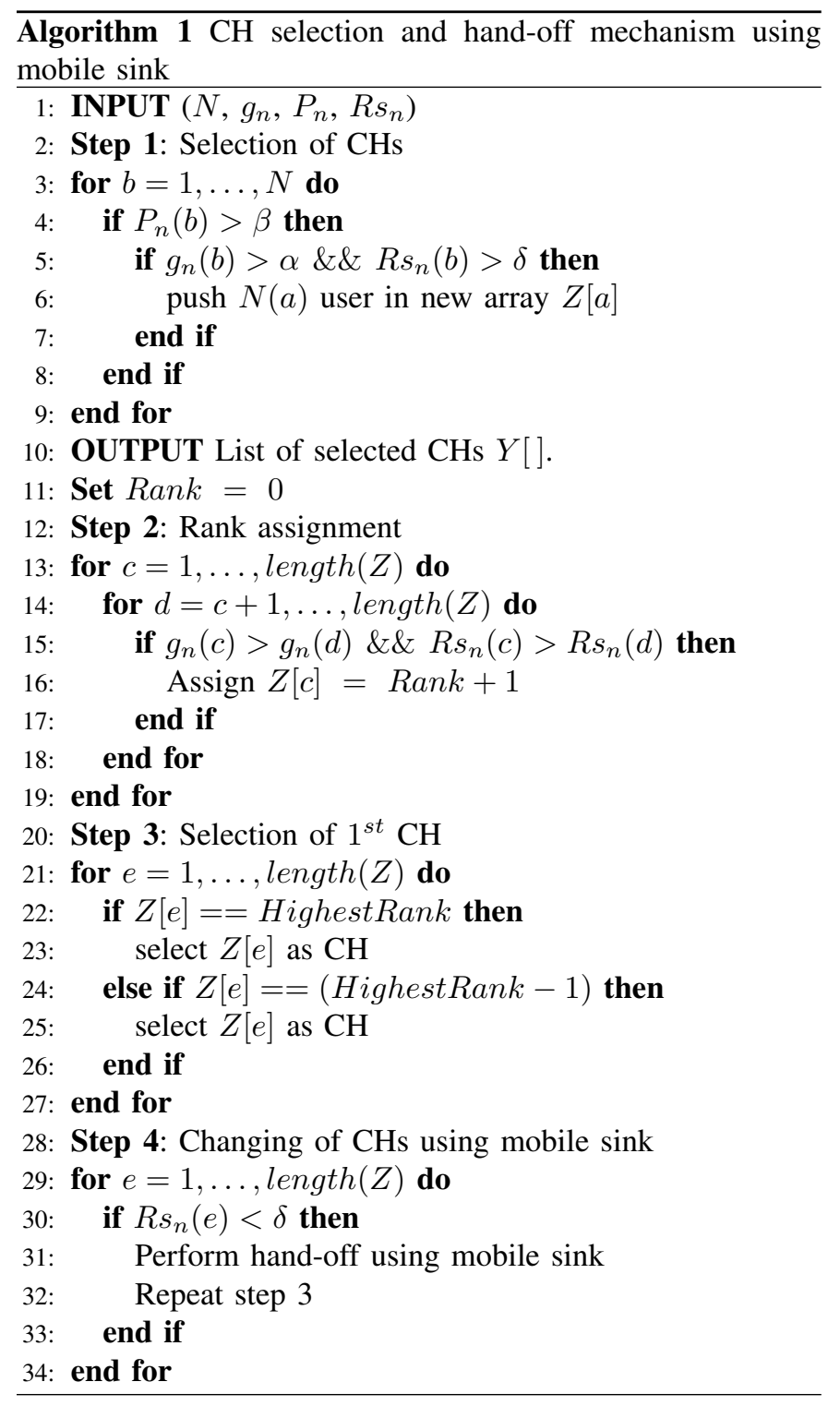

\section{PERformance Evaluation}

In this section, we demonstrate the improvement achieved regarding the energy efficiency of WSN using some simulation performed in MATLAB. We use two performance indicator parameters, i.e. lifetime and the average residual energy of WSN, respectively, to show the significance of our proposed scheme. Furthermore, we compare our proposed technique with a state-of-art dynamic $\mathrm{CH}$ selection mechanism available in the literature.
TABLE II

SiMULATION PARAMETERS

\begin{tabular}{c|l}
\hline \hline Parameter & \multicolumn{1}{c}{ Value } \\
\hline$\delta$ & 2.5 Joule \\
\hline$\beta$ & $-120 \mathrm{dBm}$ \\
\hline$\gamma$ & $-120 \mathrm{dBm}$ \\
\hline$P t_{n}$ & $8 \mathrm{dBm}$ \\
\hline$N$ & 50 to 500 \\
\hline round & 200 seconds \\
\hline$d$ & 2 meters \\
\hline$\tau$ & 3.5 \\
\hline Powerbank & 10 Joule \\
\hline \hline
\end{tabular}

\section{A. Simulation Parameters}

In the experimental setup a total number of $N=50 \mathrm{SNs}$ are deployed in a WSN cluster. The total coverage area of a WSN cluster is set as $300 \mathrm{~m}^{2}$. The maximum transmit power $\mathrm{Pt}_{n}$ for each $\mathrm{SN}$ is set as $8 \mathrm{dBm}$, and it varies between $1 \mathrm{dBm}$ and $8 \mathrm{dBm}$ in discrete levels, depending upon their residual energy. The value of path loss exponent $\tau$ is set as 3.5. We assumed that at the deployment stage, each SN is equipped with a power bank of 10 Joules and it decreases by 0.5 Joule with packet transmission. The SNR threshold $\gamma$ is set as $-120 \mathrm{dBm}$ and the channel gain threshold $\alpha$ correlates with $\gamma$. The hand-off threshold $\delta$ strictly depends upon the remaining power in the power bank of each SN and is set as 2.5 Joule. The SNs is equipped with a single antenna for wireless transmission, and an energy harvesting circuit is installed on it. The selected CHs that are in sleep mode and to be renominated for the selection of $\mathrm{CH}$, can perform energy harvesting, while remaining SNs are restricted. The BS is located at distance $d=2$ meters from the WSN cluster as shown in Fig. 1. We have also assumed that each SN transmits its packet to the BS, by using its allocated time slot, while the $\mathrm{CH}$ can aggregate up to $5 \mathrm{SNs}$ packets before transmitting it to the BS. The value of a single round is set at 200 seconds. We have assumed that the SNs performing energy harvesting can charge up their battery by 0.25 Joules in a single round. Moreover, the numerical values considered during the simulation process is summarized in Table II.

\section{B. Simulation Results}

In this section, we have compared our proposed technique with [19], using some key parameters, i.e. lifetime and average residual energy of WSN. Firstly, in Fig. 2 we have plotted the lifetime of WSN against the varying number of SNs in a WSN cluster. For this experimental setup we have varied the value of $N$ from $N=50$ to $N=300$. From the simulation results, it is quite evident that our proposed scheme outperforms and increases the overall lifetime of a WSN by $33 \%$ in comparison to [19]. The increment in the lifetime of a WSN by using our proposed approach can be explained from the fact that, our approach uses a group of $\mathrm{CHs}$ rather than sticking to a single $\mathrm{CH}$. Moreover, the scheme in [19] looks for a new $\mathrm{CH}$ after 


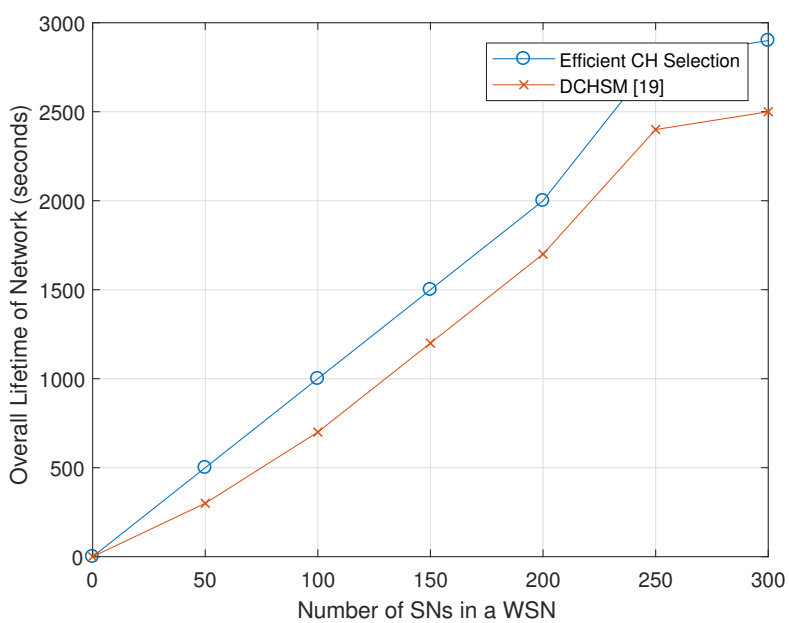

Fig. 2. A comparison of overall lifetime with varying number of SNs in a WSN cluster.

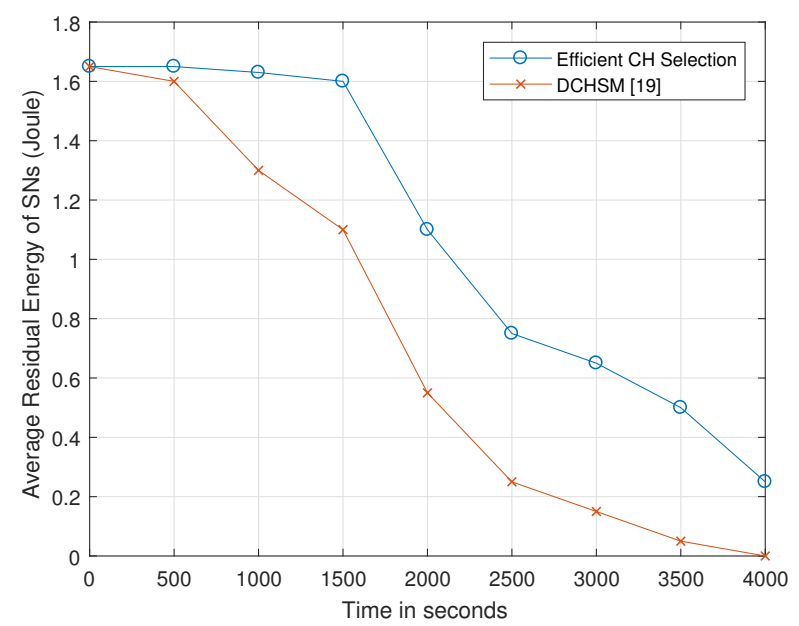

Fig. 3. A comparison of overall average residual energy of a WSN cluster with varying number of rounds during $T$ time duration.

the death of the first $\mathrm{CH}$ and does not propose any method to reuse that $\mathrm{CH}$. Finally, the concept of using a mobile sink makes the hand-off stage more efficient without the loss of extra energy and reduces the delay.

In Fig. 3, we have shown a trend of residual energy with some successful rounds. Our proposed scheme shows a significant improvement, by stabilizing the residual energy even with an increased number of rounds. In comparison to a state-of-art methodology [19], our proposed scheme enhanced the overall average residual energy by the orders of 1.5 in magnitude. The stability in the average residual energy can be explained by the utilization of energy harvesting by the CHs. Furthermore, the decreased performance of [19] is because it drains all the energy of the current $\mathrm{CH}$. Moreover, in [19], the mechanism of redundant SNs can be considered as an efficient method but on the expense of increased cost.

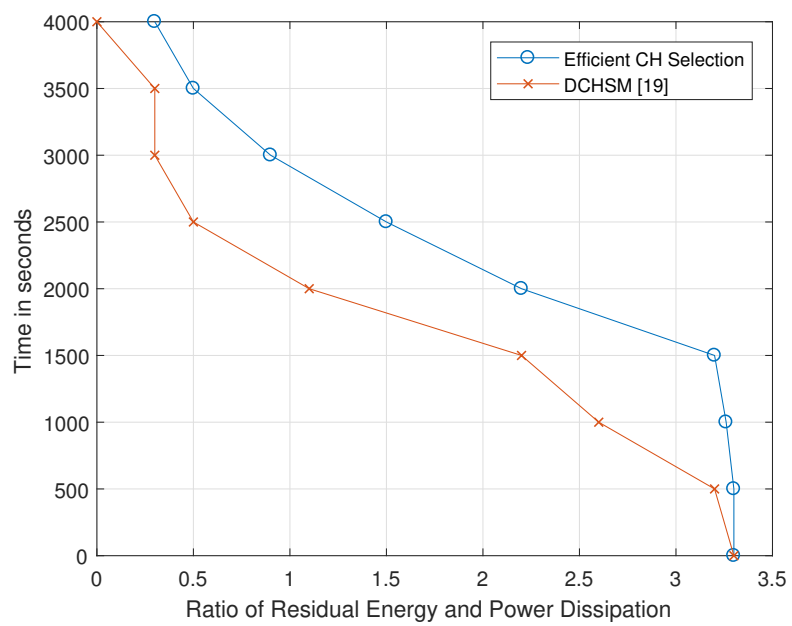

Fig. 4. A comparison of ratio of average residual energy and power dissipation per round with the total number of rounds taken during $T$ time duration.

To further show the improvement in overall performance of a WSN by using our proposed scheme, we have plotted the variation in the ratio of average residual energy and power dissipated per round, with the total number of successful rounds during the $T$ time duration. This variation can be seen in Fig. 4. For each successful round, the average residual energy of a WSN is divided by the total power dissipated by the WSN during packet transmission. In comparison to [19], our algorithm shows an increase in improvement by order of approximately 2.5 in magnitude. The improvement is by the utilization of energy harvesting performed by the SNs, competing to renominated as CHs. Moreover, the trend is to some extent similar in both the schemes. The incorporation of the energy harvesting circuit in our scheme also increases the cost, but in return, it increases the overall average residual energy and lifetime of a WSN.

\section{CONCLUSION}

In this paper, an energy efficient mechanism for the selection of $\mathrm{CHs}$ of WSNs is presented. The experimental results are carried out by considering the average residual energy and the lifetime of a WSN. The comparison has been made with a state-of-art technique available in the literature, to validate the performance of our proposed algorithm. The proposed scheme improves the inequality of average energy consumption and reduces the delay effect in the transmission by using a mobile sink during the hand-off stage, which prolongs the WSN lifetime. Furthermore, our proposed scheme enables the reuse of a $\mathrm{CH}$ node by utilizing the energy harvesting technique. Finally, the proposed scheme shows an improvement in the lifetime of a WSN by a factor of $33 \%$ and the average residual energy has been increased by 1.5 orders of magnitude while comparing with the DCHSM method. In future, we are aiming to enhance the re-usability of $\mathrm{CHs}$ by employing some machine learning techniques. 


\section{ACKNOWLEDGMENT}

This work was supported in part by the National Nature Science Foundation of China under grants 61471157, the Fundamental Research Funds for the Central Universities under grant 26120182018B23014 and ESPRC UK Global Challenges Research Fund (GCRF) allocation under grant number EP/P028764/1.

\section{REFERENCES}

[1] S. Isik, M. Y. Donmez, and C. Ersoy, "Cross layer load balanced forwarding schemes for video sensor networks," Ad Hoc Networks, vol. 9, no. 3, pp. 265-284, 2011.

[2] W.-Y. Chung, B. G. Lee, and C. S. Yang, "3d virtual viewer on mobile device for wireless sensor network-based rssi indoor tracking system," Sensors and Actuators B: Chemical, vol. 140, no. 1, pp. 35-42, 2009.

[3] B. Hu, W. Chen, and J. Zhou, "High performance flexible sensor based on inorganic nanomaterials," Sensors and Actuators B: Chemical, vol. 176, pp. 522-533, 2013.

[4] H. Yang and Y. Zhang, "A study of supercapacitor charge redistribution for applications in environmentally powered wireless sensor nodes," Journal of Power Sources, vol. 273, pp. 223-236, 2015.

[5] A. Alphonsa and G. Ravi, "Earthquake early warning system by IoT using wireless sensor networks," in Wireless Communications, Signal Processing and Networking (WiSPNET), International Conference on, pp. 1201-1205, IEEE, 2016.

[6] I. E. Commission et al., Internet of things: wireless sensor networks. IEC, 2014.

[7] L. A. Grieco, G. Boggia, S. Sicari, and P. Colombo, "Secure wireless multimedia sensor networks: a survey," in 2009 Third International Conference on Mobile Ubiquitous Computing, Systems, Services and Technologies, pp. 194-201, IEEE, 2009.

[8] S. Kumar, H. Lim, and H. Kim, "Energy optimal scheduling of multichannel wireless sensor networks for wireless metering," in Electronics, Information, and Communications (ICEIC), 2016 International Conference on, pp. 1-5, IEEE, 2016.

[9] M. A. Jamshed, O. Amjad, and E. Zeydan, "Multicore energy efficient scheduling with energy harvesting for wireless multimedia sensor networks," in Multi-topic Conference (INMIC), 2017 International, pp. 1-5, IEEE, 2017.

[10] C. Wang, Y. Zhang, X. Wang, and Z. Zhang, "Hybrid multihop partitionbased clustering routing protocol for WSNs," IEEE Sensors Letters, vol. 2 , no. 1, pp. 1-4, 2018.

[11] Y. Zou and K. Chakrabarty, "Sensor deployment and target localization based on virtual forces," in INFOCOM 2003. Twenty-Second Annual Joint Conference of the IEEE Computer and Communications. IEEE Societies, vol. 2, pp. 1293-1303, IEEE, 2003.

[12] D. He, N. Kumar, J. Chen, C.-C. Lee, N. Chilamkurti, and S.-S. Yeo, "Robust anonymous authentication protocol for health-care applications using wireless medical sensor networks," Multimedia Systems, vol. 21, no. 1, pp. 49-60, 2015.

[13] S. Poduri and G. S. Sukhatme, "Constrained coverage for mobile sensor networks," in Robotics and Automation, 2004. Proceedings. ICRA'04. 2004 IEEE International Conference on, vol. 1, pp. 165-171, IEEE, 2004.

[14] S. Hosseinirad, M. Alimohammadi, S. Basu, and A. Pouyan, "Leach routing algorithm optimization through imperialist approach," International Journal of Engineering, Transactions A: Basics, vol. 27, no. 1, pp. 39-50, 2014.

[15] O. Younis and S. Fahmy, "HEED: a hybrid, energy-efficient, distributed clustering approach for Ad-hoc sensor networks," IEEE Transactions on mobile computing, vol. 3, no. 4, pp. 366-379, 2004.

[16] J. R. Srivastava and T. Sudarshan, "A genetic fuzzy system based optimized zone based energy efficient routing protocol for mobile sensor networks (OZEEP)," Applied Soft Computing, vol. 37, pp. 863-886, 2015.

[17] W. Ke, O. Yangrui, J. Hong, Z. Heli, and L. Xi, "Energy aware hierarchical cluster-based routing protocol for WSNs," The Journal of China Universities of Posts and Telecommunications, vol. 23, no. 4, pp. 46-52, 2016.
[18] S. Tyagi and N. Kumar, "A systematic review on clustering and routing techniques based upon LEACH protocol for wireless sensor networks," Journal of Network and Computer Applications, vol. 36, no. 2, pp. 623645,2013

[19] D. Jia, H. Zhu, S. Zou, and P. Hu, "Dynamic cluster head selection method for wireless sensor network," IEEE Sensors Journal, vol. 16, no. 8, pp. 2746-2754, 2016.

[20] M. Rayenizadeh, M. K. Rafsanjani, and A. B. Saeid, "Cluster head selection using hesitant fuzzy in wireless sensor networks," in 2018 6th Iranian Joint Congress on Fuzzy and Intelligent Systems (CFIS), pp. 139-141, IEEE, Feb 2018.

[21] K. A. Darabkh and L. Al-Jdayeh, "A new fixed clustering based algorithm for wireless sensor networks," in 2018 14th International Wireless Communications Mobile Computing Conference (IWCMC), pp. 71-76, IEEE, June 2018.

[22] L. Gao and Z. Li, "Energy consumption balance cluster-head selection algorithm for Wireless Sensor Network," in 2013 International Conference on Computer Sciences and Applications, pp. 179-182, IEEE, Dec 2013.

[23] M. Hongbin, W. Yingli, Y. Shuang, Y. Hai, and L. Zhenhai, "Hybrid key management mechanism based on double cluster head structure," in 2012 Second International Conference on Instrumentation, Measurement, Computer, Communication and Control, pp. 1164-1167, IEEE, Dec 2012. 